\title{
Heteroisotopic molecular behavior. The Valence-Bond Theory of the Positronium Hydride
}

\author{
Flávia Rolim, Tathiana Moreira, and José R. Mohallem \\ Laboratório de Átomos e Moléculas Especiais, Departamento Física, ICEx, \\ Universidade Federal de Minas Gerais, PO Box 702, 30123-970, Belo Horizonte, MG, Brazil
}

Received on 10 December, 2003

\begin{abstract}
We develop an adiabatic valence-bond theory of the positronium hydride, HPs, as a heteroisotopic diatomic molecule. Typical heteronuclear ionic behaviour comes out at bonding distances, yielded just by finite nuclear mass effects, but some interesting new features appears for short distances as well.
\end{abstract}

\section{Introduction}

In theory of diatomic molecules, the concepts of homo and hetero nuclearity have been developed in a natural way, to recognize whether a molecule is made up of equal or different atoms, respectively. Since atoms differ from each other by their atomic charge $Z$, which is contained in the potential energy part of the Hamiltonian operator, the BornOppenheimer (BO) theory of molecules [1], based on a clamped-nuclei electronic Hamiltonian, is sufficient to account for these features and to explore all the consequences of point group symmetries displayed by the electronic wavefunctions. Any further features yielded by coupling of electronic and nuclear motion are treated as non-adiabatic effects, whose calculations involve many BO states [1]. For polyatomic molecules, the concept and terminology of "equivalent atoms" are introduced, meaning units of the same chemical element that transform among each other by symmetry operations (for example, the two hydrogen atoms in the water molecule).

On the other hand, recent developments have shown that a variational adiabatic (non-BO) approach for the electronic problem is able to account for another property of atoms, their mass $M$ (the isotopic effect), in a somewhat analogous way of $Z$ [2]-[6]. This introduces the idea of homo and heteroisotopic molecular behavior. For example, being D the symbol for deuterium, $\mathrm{H}_{2} \mathrm{O}$ and $\mathrm{D}_{2} \mathrm{O}$ are homoisotopic but HDO is heteroisotopic, the last one having its symmetry broken from $C_{2 v}$ to $C_{s}$ [6]. The consequent isotope shifts, dipole moments and new spectroscopical transitions, are real and measurable [7].

This new way of symmetry classification of molecules may look strange, however, as it is generated by kinetical terms of the total Hamiltonian, instead of by a rigid framework of nuclear charges. Furthermore, for typical molecules, the effects are too small to be visualized in electronic density maps, as usually done in the common homo and heteronuclear cases. The theoretical signature of symmetry breaking is just the non-commutability of some symmetry operators with the adiabatic Fock operator [6].

Fortunately, there is the singular case of the positronium hydride, HPs $\left(\mathrm{H}=\mathrm{H}^{+} e^{-}+\mathrm{Ps}=e^{+} e^{-}\right)$, which can be seen as an extreme homonuclear but heteroisotopic isotopomer of $\mathrm{H}_{2}$. The large difference between the proton and positron masses yields a considerable asymmetry, that stimulates us to investigate how it affects the electronic distribution, in comparison with the common heteronuclear case (which we call here the $Z-M$ analogy). As a matter of fact, Saito [8] has already pointed out that the electronic density of HPs, obtained from four-body correlated calculations, shows a visual approximate molecular behavior, but this kind of approach can give no further structural details, however. We are, on the other hand, interested in exploring possible $Z-M$ analogy effects with a theory in which the "nuclear" (proton and positron) and electronic motions are adiabaticaly separated, that is, with the same theoretical treatment used with standard isotopomers, in order to better understand their isotopic properties.

It is well known that the valence-bond (VB) theory is appropriate to analyse in detail the bonding of a typical diatomic molecule. Particularly for a heteronuclear one, a mixed covalent-ionic behavior of the wavefunction points to a polarization of the electronic distribution toward the more electronegative atom, measured by the relative value of the linear coefficient of the ionic structure. Furthermore, the exponents of the atomic orbitals measure the effective charge of each nucleus felt by the electrons and become indicative of the same above effect, as well. Classical chemical concepts as, for example, electronegativity, have immediate interpretation in terms of the VB output.

Here, we check whether a VB calculation of HPs is able to study the consequences of its mass asymmetry on the electron distribution and deeply explore the $Z-M$ analogy. This is done in the following section.

\section{The valence-bond theory of HPs}

\subsection{Methodology}

Atomic units (au) and conventional notation are used throughout. Our approach to include the finite nuclear mass effects in molecular electronic structure calculations [2]-[4] 
has been to look for electronic wavefunctions $\Phi_{k}$ that are eigenfunctions of the total Hamiltonian, instead of the BO one, that is,

$$
H \Phi_{k}=\epsilon_{k} \Phi_{k}
$$

Different from the common adiabatic approximation [9], that corrects just the electronic energy (or the potential energy curves, PEC), this approach allows the electronic wavefunctions to account for the nuclear motion as well. A product wavefunction is assumed for any state $k$, that is, $\Psi_{k}=\Phi_{k} \chi_{k}$, where $\chi$ is a nuclear wavefunction. The solution of equation (1) is done in a variational sense, which explains the terminology "variational adiabatic". Further approximations are explained bellow.

The system, $A B e^{-} e^{-}$, is treated as being heteroisotopic, which means here that the positive nuclei $A\left(\mathrm{H}^{+}\right)$and $B\left(e^{+}\right)$, separated by the distance $R$, have different masses, $M_{A}$ and $M_{B}\left(M_{B}=1\right.$ ua), but the same charge, $Z=+1$ au.

For the definition of our electronic basis, we consider a covalent (Heitler-London, HL), and two ionic VB structures, so that a spatial symmetric state can be written as the superposition

$$
\begin{gathered}
\Phi_{k}=c_{k 1}[a(1) b(2)+a(2) b(1)] \\
+c_{k 2} a(1) a(2)+c_{k 3} b(2) b(2) .
\end{gathered}
$$

Here, $a$ and $b$ are normalized $1 s$ orbitals centered on nuclei $A$ and $B$, with exponents $\zeta_{A}$ and $\zeta_{B}$, respectively, being all the linear and non-linear parameters dependent on $R$. To save notation, we refer, in what follows, to the HL, $A$-ionic and $B$-ionic structures, with obvious meaning, and call this set the VB basis.

We first attempt to solve equation (1) to obtain the PECs

$$
U_{k}(R)=\epsilon_{k}(R)+\frac{1}{R} .
$$

On this level, the problem has been treated, in our laboratory, within a modified-electron-mass approach [2],[3], which works well for one and two-electron homonuclear diatomic molecules. For more complicated systems we resorted to an empirical correction [4] for which a model Hamiltonian has been developed later [10]. Summarizing the prescription of ref. [4], first assume that for an electron occupying the atomic orbital $a$ centered on a nucleus $A$ with mass $M_{A}$, the finite nuclear mass correction (FNMC) to its energy is (unormalized),

$$
Q_{A}=\frac{\left\langle a(1)\left|-\nabla_{1}^{2}\right| a(1)\right\rangle}{2 M_{A}} .
$$

This correction is exact for an isolated non-relativistic hydrogenic atom. For a polyatomic molecule, the FNMC is accomplished by considering the $\mathrm{BO}$ hamiltonian in the standard form $H_{B O}=\sum_{i} h(i)+\frac{1}{r_{i j}}$, where the $h(i)$ are one-electron hamiltonians, and obtaining its matrix elements in the electronic basis. Wherever a term like $\langle a|h| a\rangle$ appears in any of these matrix elements, a correction like (3) is added to it, provided that terms originated by symmetrization of a VB structure contribute just once. This procedure assures that the corrections will be properly placed in the hamiltonian matrix. In the present case, the ground state (gs) correction with wavefunction (2), becomes simply

$$
\begin{aligned}
Q= & c_{1}^{2}\left(Q_{A}+Q_{B}\right)+c_{2}^{2}\left(2 Q_{A}\right)+c_{3}^{2}\left(2 Q_{B}\right) \\
& +c_{1} c_{2} Q_{A}\langle a \mid b\rangle+c_{1} c_{3} Q_{B}\langle b \mid a\rangle .
\end{aligned}
$$

Note that if the one-electron atoms are set apart, only the first term survives and the correction becomes exact. For finite $R$, the correction performs quite well, as shown in refs. [3],[4]. The same result of equation (4) could be obtained with the model Hamiltonian for the FNMC [10] as well.

The atomic orbitals and the linear coefficients are optimized in order to minimize the adiabatic electronic energy,

$$
\epsilon_{k}(R)=\epsilon_{B O_{k}}(R)+Q_{k}(R),
$$

which yields the PECs for each state $k$. The results of equations (4) and (5) express our approximate solution of equation (1).

\subsection{The VB basis}

Before concerning the complete expansion of the wavefunction in the VB basis, it is interesting to verify how this nonorthogonal basis can be devised. Our treatment resembles an old one by Zener [11] for ionic molecules, but with some additional complications due to the need of variational determination of each state (see bellow). We ignore for while the $B$-ionic structure, since, in view of the smaller energy of the corresponding dissociation products, $\mathrm{H}^{+}+\mathrm{Ps}^{-}$, it must not correspond to a low-lying state of the system. On the contrary, the HL structure and the $A$-ionic structure are expected to correlate to lower states of HPs.

With just the HL structure as the electronic wavefunction, the FNMC (4) becomes $Q=Q_{A}+Q_{B}$, and a variational calculation of the PEC yields curve 1 of Fig. 1A. It advances the dissociation of the system in $\mathrm{H}+\mathrm{Ps}$, giving the exact separated-atom (SA) energy, $E(\infty)=-0.74973$ au. With the $A$-ionic structure, the correction is $Q=2 Q_{A}$ and curve $\mathbf{2}$ is obtained, which advances the dissociation in $\mathrm{H}^{-}+\mathrm{Ps}^{+}$, with the independent particle approximation energy of $E(\infty)=-0.53858$ au. Curve 1 must thus correlate better to the electronic ground state. As a matter of fact, both curves, corresponding to non-orthogonal states, mimic the ground state, for intermediate values of $R$. In the united-atom (UA) limit, the HL structure tend to imitate the ionic one, which is far more appropriate to this limit, so that the curves close approach and cross (not an actual crossing, however).

This study justifies our chosen VB basis. It advances two equally important structures, the HL being the one that must dominate the wavefunction at large distances, the $A$-ionic being dominant at small distances and the mixing of them accounting for most of the bonding features. The $B$-ionic structure, centered on $e^{+}$, is included for the sake of completeness of our description and to increase the variational character of the wavefunction. 

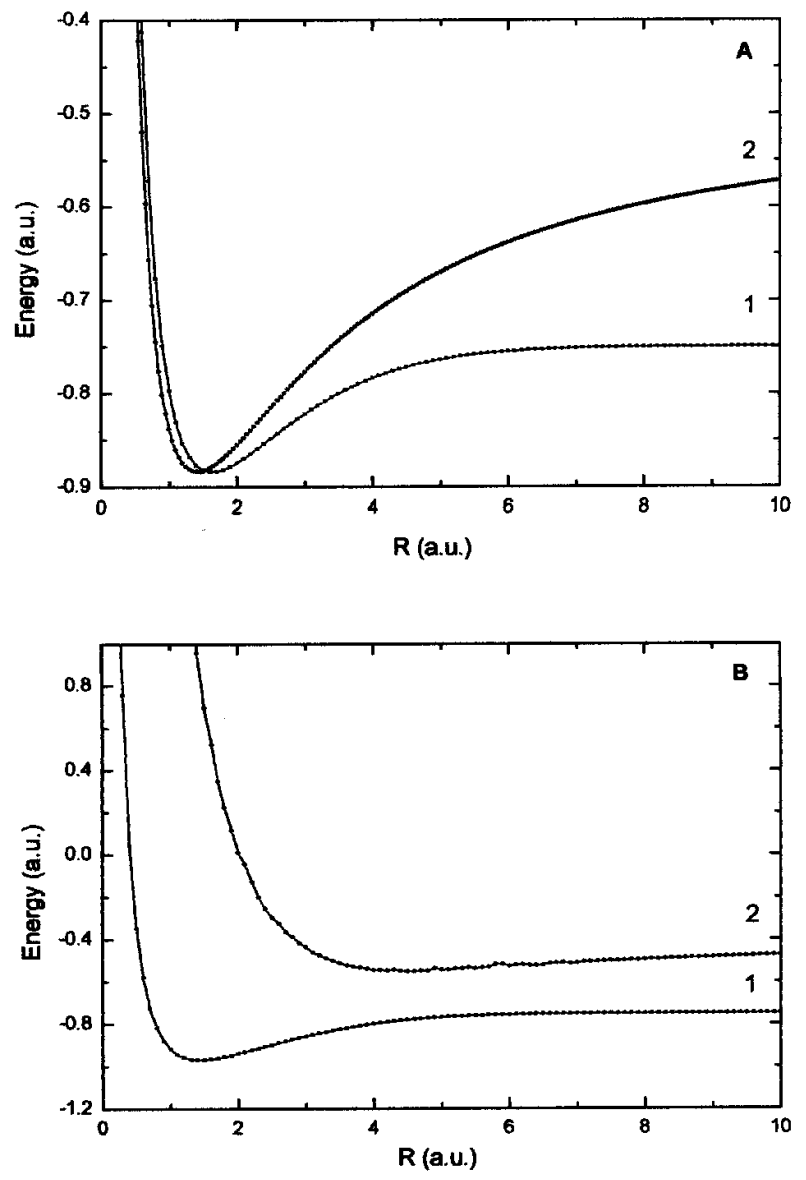

Figure 1. A- Potential energy curves for the 1) covalent and 2) $A$ ionic electronic states of HPs, B- Potential energy curves for the 1) ground bonding and 2) first excited state of HPs.

In some extent, this behavior of the VB basis is analogous to that reported for typical ionic molecules [11],[12], in which no effects of nuclear motion are considered, however. In spite the two atoms having the same nuclear charge and just one electron each, the mass effects advances the $A$-ionic structure as fundamental in the description of the electronic gs of HPs.

\subsection{Calculations with the full VB basis}

We next diagonalize the hamiltonian in the full VB basis, obtaining three electronic states $\Phi_{k}$, each one generating a PEC. They are obtained with simultaneous optimization of the parameters $c_{k 1}, c_{k 2}, c_{k 3}, \zeta_{A}$ and $\zeta_{B}$ for each value of $R$, to get the minimum of the gs PEC at each $R$. An immediate effect is the uncrossing of the first two lower curves, shown in Fig. 1B. The lower curve becomes the bonding ${ }^{1} \sum_{g}$ state of HPs, and the second correlates with higher states having $\mathrm{H}^{-}$as a dissociation product (there are other states of intermediate energy between them, corresponding to orbital excitations of the $\mathrm{H}$ and $\mathrm{Ps}$ atoms in the SA limit).

We then solve the nuclear equation,

$$
\left[-\frac{\nabla_{R}^{2}}{2 \mu_{A B}}+U_{k}(R)-E\right] \chi_{k}(R)=0,
$$

for the bound state nuclear wavefunction $\chi_{1}(R)$ and the corresponding energy $E_{1}$. Although the minimum of the PEC is around $1.3 \mathrm{au}$, we cannot speak here of an equilibrium position, due to positron delocalization. The nuclear wavefunction displays this feature, spreading on a larger range of distances in comparison with $\mathrm{H}_{2}$, and lead to an average proton-positron distance of $\langle R\rangle=3.34$ au. In view of this behavior, we consider the bonding distance as lying in the range $3 \leq R \leq 4$ au.

The energy for the $S$ bound state of HPs is $E=$ -0.7889 au [13]. We obtained $E_{1}=-0.7668$ au with the reduced mass of the nuclei $\left(\mu_{A B}\right)$, and $E_{1}=-0.8070$ au with the empirical reduced mass of the atoms $\left(\mu_{A B}^{a t}=\right.$ $\left.\left(\frac{1}{M_{A}+1}+\frac{1}{M_{B}+1}\right)^{-1}\right)$. Some researchers have pointed a connection between non-adiabatic effects and a reduced mass variable with $R$ [14],[15],[9],[16]. When we use an empirical average reduced mass defined as

$$
\mu_{A B}^{a v e}=\frac{c_{1}^{2} \mu_{A B}^{H L}+c_{2}^{2} \mu_{A B}^{i o n A}+c_{3}^{2} \mu_{A B}^{i o n B}}{c_{1}^{2}+c_{2}^{2}+c_{3}^{2}}
$$

with $\mu_{A B}^{H L}=\mu_{A B}^{a t}, \quad \mu_{A B}^{i o n A}=\left(\frac{1}{M_{A}+2}+\frac{1}{M_{B}}\right)^{-1}$, etc., we get a very reasonable energy of -0.7879 au. For the pair annihilation rate, calculated with the formula $\Gamma=-50.47\langle\Psi| \delta\left(\vec{r}_{e^{+}}-\vec{r}_{e^{-}}|\Psi\rangle\right.$ [17], we get $2.42 \times 10^{9} \mathrm{~s}^{-1}$, which compares well with the accepted value of $2.32 \times 10^{9} s^{-1}$ [18]. However, the point here is not to get accurate calculations of properties, which could not be attained with our näive wavefunction anyway. These results serve only to lend reliability to the $Z-M$ analogies we are looking for.

The expansion coefficients, shown in Fig. 2, confirm the mixed covalent-ionic behavior of the ground and excited electronic states. Particularly for the gs (coefficients $c_{k 1}$ ), we note the expected predominance of the $A$-ionic structure at small distances and of the covalent one at large distances, both of them being important at bonding distances, with close analogy with the typical ionic molecule case. On the other hand, the coefficient of the $B$-ionic structure is very small for any value of $R$. This feature is not a surprise since we do not expect the cluster $e^{-} e^{+} e^{-}$(stable, with energy of $-.262 \mathrm{au}$ ) to be relevant in the bonding of HPs.

Another interesting outcome is the behavior of the orbital exponents, or effective charges, for the ground state, Fig. 3. They are measures of the size of the atomic orbitals and of screening effects. The exponent $\zeta_{B}$ of the isolated Ps atom is already half of the $e^{+}$charge, due to reduced mass effects. The orbital $a$, with exponent $\zeta_{A}$, presents an analogous variation with $R$ as for $\mathrm{H}_{2}$ [19]. From large values until $R \gtrsim 2$ ua, $\zeta_{B}$ shows a regular behavior as well. We can identify the effective charges around 0.9 au for the proton and 0.6 au for the positron (at large distances they are exactly 1 . and 0.5 respectively, the last one already including reduced mass effects). In spite the real charges being both +1.0 , the mass effects impose a behavior of the effective charges, mainly $\zeta_{B}$, analogous to what happens in a typical ionic molecule, with $\zeta_{A}>\zeta_{B}$. 

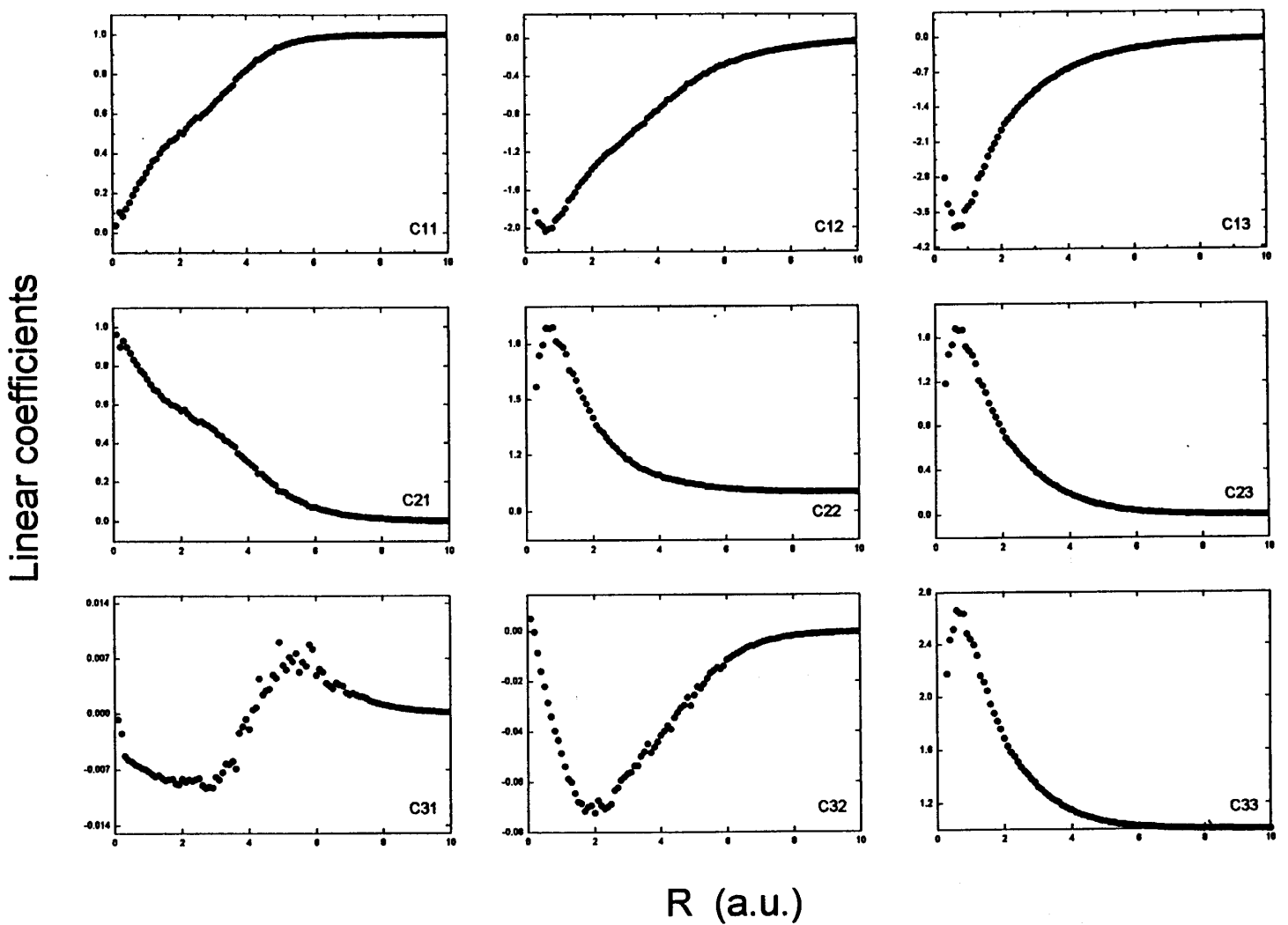

Figure 2. Linear coefficients, in relative units, versus nuclear distance. Note that the vertical scales are not necessarily the same.



Figure 3. Orbital exponents versus nuclear distance. In the inset, the atomic orbital $b$ for some marked values of the nuclear distance. The circles enclose $90 \%$ of the electronic probability. 
For smaller values of $R$, on the other hand, the behavior of $\zeta_{B}$ becomes singular. Close to $R=3.0$ au it starts growing up critically, before falling down to the expected UA limit. The explanation is that the corresponding strong contraction of the orbital $b$ centered on $e^{+}$, illustrated in the inset of Fig. 4, is necessary in order to the positron to cross the electronic cloud of the $\mathrm{H}$ atom and the electrons to change their motion, and adapt to the orbital of the composite nucleus $\mathrm{H}^{+}+e^{+}$. In this process, the system must change its dimensional scale, from the scale of an exotic diffuse positronic molecule to that of a typical atom. This behavior seems to have no analog in any other typical ionic molecules, and is a major difference encountered between HPs and typical systems (in fact, a test on $\mathrm{HeH}^{+}$did not show such effect). This is a singular consequence of the asymmetric behavior of the electronic distribution of HPs being caused by nuclear mass differences, instead of charge differences.

Most illustrative results are the graphs of the electronic densities on the molecular axis, shown in Fig. 4, and the corresponding contour maps in a plane that contains the axis, in Fig. 5, for some values of $R$. They give an idea of the dynamical formation of the system from the constitutive atoms. The electronic densities show a typical chemical bonding profile, with well defined peaks on the nuclei and a valley between them. The contour maps are shown for different situations, from the SA to the UA limits. The $R=3.0$ map is typical of the HPs bonding distance. The $R=.25$ map corresponds to the more extreme contraction of orbital $b$. For $R=0.1$ it seems that the system had almost become the UA atom, with spherical symmetry. With due care to the different scales of the figures, the reader can note the dimensional change from the exotic molecule to the UA typical atom (realize also that the real dimension is inversely proportional to the dimension of the pictures). For distances around $R=1.0$ au, the contour maps are analogous of those of diatomic molecules with disparate nuclear charges [20]. These kind of molecules present a strong ionic behavior reflected in a large contribution of the ionic structure in the VB wavefunction. In this region, the Ps atom seems to loose its identity in the complex, which suggested the next application.

\subsection{The topology of the electronic density}

A modern analysis of molecular structure is the topological study of the electronic density, developed mainly by Bader and collaborators [20]. The gradient field of the electronic density $\rho$ is characterized by regions (basins) separated by surfaces of $\vec{\nabla} \rho=0$. These basins are identified as "topological atoms". We use this technique to investigate whether the positronium atom keeps its identity in the HPs complex, for different values of $R$. The gradient of the gs electronic density $\vec{\nabla} \rho=\vec{\nabla} \Phi_{1}^{*} \Phi_{1}$, with electronic wavefunction (2), is easily obtained in elliptical coordinates, yielding the graphs in Fig. 6. Once again, typical heteronuclear behavior comes out at bonding distances, with the topological Ps atom occupying a smaller basin than the topological $\mathrm{H}$ atom, shown in the $R=3.6$ au graph. In this kind of analysis, a signature of chemical bonding is the appearance of a critical point between atoms $A$ and $B$, which is present in our system from $R=3.0$ au to much larger values. For $R$ smaller than this value, corresponding to the region where the Ps orbital start contracting, the critical point disappear, which constitutes a further indication that for these distances the system has a singular behavior, and seems to mean that the topological Ps does not exist anymore. This is expressed in the $R=1.45$ au graph, where no critical point appears and no clear division of the basins can be devised between the nuclei.

\section{Further discussion}

The variational adiabatic approximation to the molecular problem introduces the finite nuclear masses into the electronic Hamiltonian. The mass asymmetry is able to generate symmetry breaking phenomena analogous to charge asymmetry. This considerations lead us to the concepts of homo and heteroisotopic molecules (accordingly, we might be tempted to propose the replacement of the usual terms "homo and heteronuclear" by "homo and heteroatomic", but the terminology is already firmly established).

The results obtained here with VB theory, that show HPs somewhat as an ionic molecule in the bonding region, impose a modification in the way we face the very concept of molecular structure. In a sense, it seems that we should decouple the symmetry properties of the electronic distribution from the idea of a rigid nuclear geometry, since these properties look the same, no matter they are established by charge or mass effects. On the contrary, these effects seem to be interchangeable. In atomic physics we find an analogous feature. The Hamiltonian of an one-electron atom $H=-\frac{\nabla^{2}}{2 \mu}-\frac{Z}{r}$ can be changed to the scaled form $H_{e s c}=\mu H=-\frac{\nabla^{2}}{2}-\frac{\zeta}{r}$, with $\zeta=\mu Z$, that is, a massscaled Hamiltonian describes an atom of fixed center and different charge, $\zeta$.

In view of these considerations, it becomes much easier to interpret some properties of isotopomers, as for example, the dipole moment of HD. From the BO theory, it has its source in complicated non-adiabatic couplings of electronic, vibrational and rotational states [21]. From the present point of view, the dipole moment is a consequence of the asymmetric electronic distribution, generated by finite nuclear mass effects. Other properties will be more easily interpreted on this basis as well.

\section{Acknowledgements}

Support by CNPq and Capes is acknowledge. 

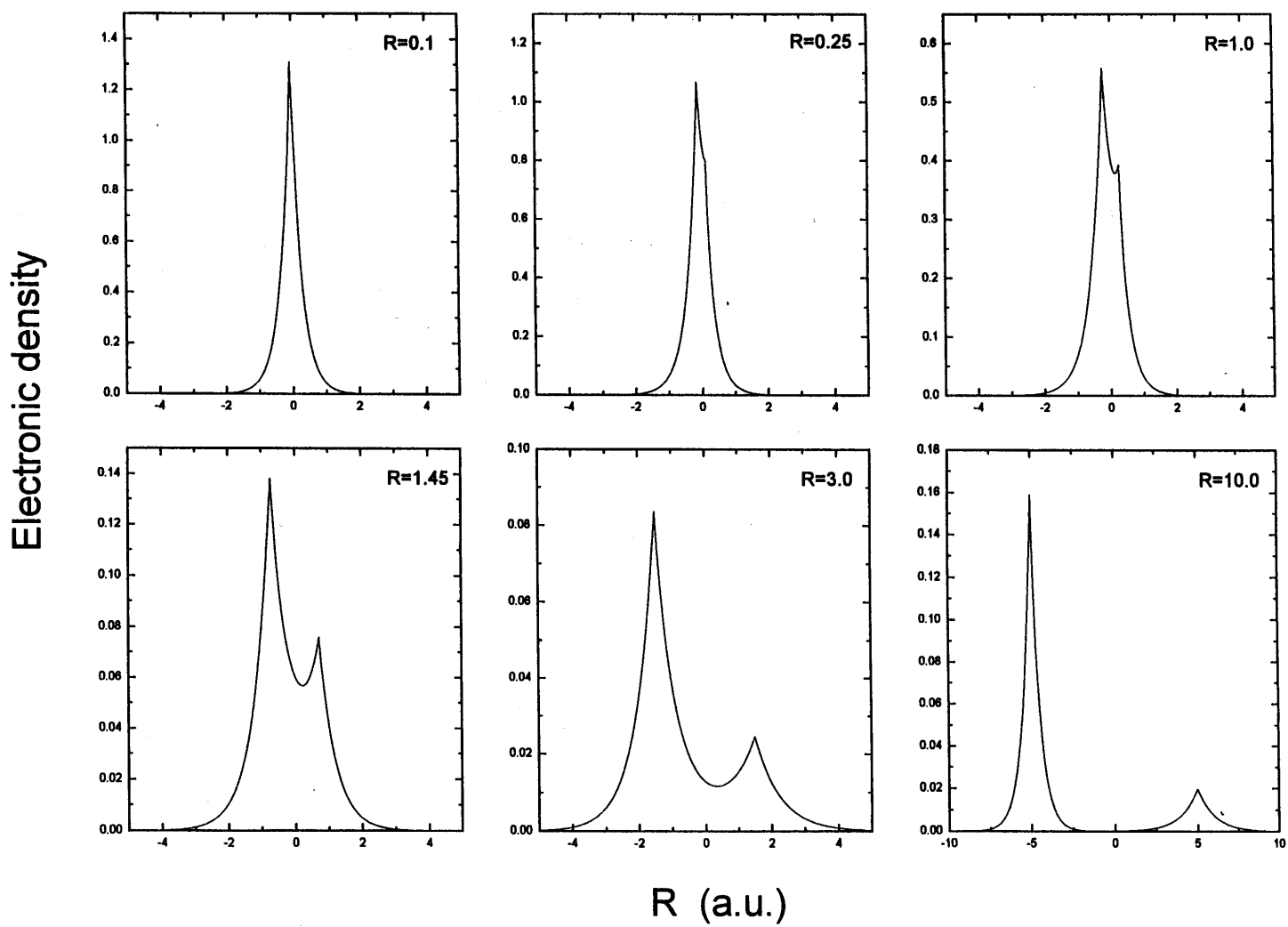

Figure 4. Electronic densities on the molecular axis for some values of the nuclear distance.
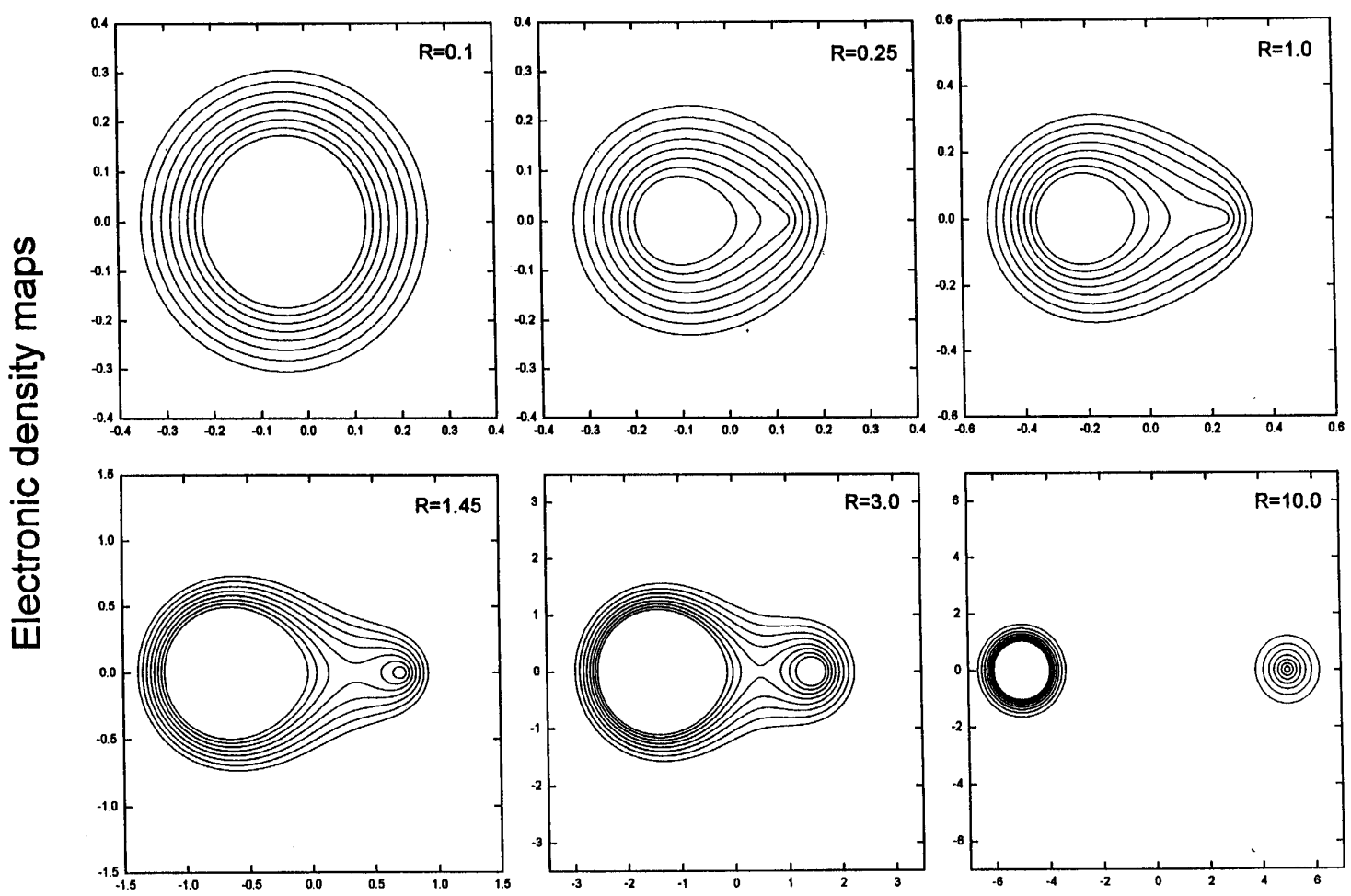

R (a.u.)

Figure 5. Maps of the electronic densities in a plane containing the molecular axis, for some values of the nuclear distance. 


\section{Gradient vector field of charge density}


Figure 6. Field lines of the gradient of the electronic density for two inter-nuclear distances. The point in black in the $R=3.6 \mathrm{au}$ curve is the critical point.

\section{References}

[1] M. Born and K. Huang, Dynamical theory of crystal lattices (Oxford University Press, New York, 1956).
[2] J. R. Mohallem, J. Phys. B: At Mol. Opt. Phys. 32, 3805 (1999).

[3] J. R. Mohallem, F. Rolim and C. P. Gonçalves, Mol. Phys. 99, 87 (2001).

[4] C. P. Gonçalves and J. R. Mohallem, Theor. Chem. Acc. 110, 367 (2003).

[5] C. P. Gonçalves and J. R. Mohallem, Chem. Phys. Lett. 367, 533 (2003).

[6] C. P. Gonçalves and J. R. Mohallem, Chem. Phys. Lett. 380, 378 (2003).

[7] A. de Lange, E. Reinhold and W. Ubachs, Int. Rev. Phys. Chem. 21, 257 (2002).

[8] S. L. Saito, Nucl Instr and Meth in Phys Res B 171, 60 (2000).

[9] See, for example, N. C. Handy and A. M. Lee, Chem. Phys. Lett. 252, 425 (1996).

[10] J. R. Mohallem, J. Mol. Struct. Theochem, in press.

[11] C. Zener, Proc. Roy. Soc. Ser. A 137, 696 (1932).

[12] R. S. Berry, J. Chem. Phys. 27, 1288 (1957).

[13] A. M. Frolov and V. H. Smith, Jr, Phys. Rev. A 55, 2662 (1997).

[14] W. Meyer, P. Botschwina, and P. Burton, J. Chem. Phys. 84, 891 (1986).

[15] R. Röhse, W. Kutzelnigg, R. Jaquet, and W. Klopper, J. Chem. Phys. 101, 2231 (1994).

[16] F. Rolim, J. P. Braga, and J. R. Mohallem, Chem. Phys. Lett. 332, 139 (2000).

[17] Chang Lee, Zh. Eksp. Teor. Fiz. 33, 365 (1958) [Sov Phys JETP 6, 281 (1958)].

[18] J. Mitroy, M.W. Bromley, and G.G. Ryzhikh, J. Phys. B: At Mol. Opt. Phys. 35, R1 (2002).

[19] J. O. Hirschfelder and J. W. Linnet, J. Chem. Phys. 18, 130 (1949).

[20] R. F. W. Bader, Atoms and Molecules, a Quantum Theory, Claredon Press, Oxford, 1995.

[21] See, for example, A. L. Ford and J. C. Browne, Phys. Rev. A 16, 1992 (1977). 